\title{
Integrated slice-specific dynamic shimming for whole-body diffusion-weighted MR imaging at 1.5 T
}

\author{
Sarah McElroy ${ }^{1}$. Jessica M. Winfield ${ }^{1}$. Olwen Westerland ${ }^{1} \cdot$ Geoff Charles-Edwards ${ }^{1}$. Joanna Bell ${ }^{1}$. \\ Radhouene $\mathrm{Neji}^{2} \cdot$ Alto Stemmer $^{3} \cdot$ Berthold Kiefer $^{3} \cdot$ Matthew Streetly $^{4} \cdot$ Vicky Goh $^{1,5}$
}

Received: 24 May 2020 / Revised: 13 November 2020 / Accepted: 17 November 2020 / Published online: 23 December 2020

(c) The Author(s) 2020

\begin{abstract}
Objective To compare integrated slice-specific dynamic shim (iShim) with distortion correction post-processing to conventional 3D volume shim for the reduction of artefacts and signal loss in $1.5 \mathrm{~T}$ whole-body diffusion-weighted imaging (WB-DWI).

Methods Ten volunteers underwent WB-DWI using conventional 3D volume shim and iShim. Forty-eight consecutive patients underwent WB-DWI with either volume shim $(n=24)$ or iShim $(n=24)$ only. For all subjects, displacement of the spinal cord at imaging station interfaces was measured on composed $b=900 \mathrm{~s} / \mathrm{mm}^{2}$ images. The signal intensity ratios, computed as the average signal intensity in a region of high susceptibility gradient (sternum) divided by the average signal intensity in a region of low susceptibility gradient (vertebral body), were compared in volunteers. For patients, image quality was graded from 1 to 5 ( $1=$ Poor, $5=$ Excellent). Signal intensity discontinuity scores were recorded from 1 to $4(1=2+$ steps, $4=0$ steps). A $p$ value of $<0.05$ was considered significant.

Results Spinal cord displacement artefacts were lower with iShim $(p<0.05)$ at the thoracic junction in volunteers and at the cervical and thoracic junctions in patients $(p<0.05)$. The sternum/vertebra signal intensity ratio in healthy volunteers was higher with iShim compared with the volume shim sequence $(p<0.05)$. There were no significant differences between the volume shim and iShim patient groups in terms of image quality and signal intensity discontinuity scores.

Conclusion iShim reduced the degree of spinal cord displacement artefact between imaging stations and susceptibilitygradient-induced signal loss.
\end{abstract}

Keywords Whole-body imaging $\cdot$ Diffusion-weighted MRI $\cdot$ Oncology $\cdot$ Artefacts $\cdot$ Echo-planar imaging

\section{Abbreviations}

DWI Diffusion-weighted imaging

EPI Echo-planar imaging

ROI Region of interest

SIR Signal intensity ratio

WB-DWI Whole-body diffusion-weighted imaging

Supplementary Information The online version contains supplementary material available at https://doi.org/10.1007/s1033 4-020-00898-6.

Sarah McElroy

sarah.mcelroy@kcl.ac.uk

Extended author information available on the last page of the article

\section{Introduction}

Whole-body MRI is accepted as the gold-standard imaging modality for suspected and newly diagnosed myeloma and plasmacytoma, and is recommended by the International Myeloma Working Group and the National Institute for Health and Care Excellence [1, 2]. Whole-body diffusion-weighted imaging (WB-DWI) was first introduced by Takahara et al. [3], and many studies have since shown the added benefit of this technique for diagnosis, staging and treatment response in oncology [4-6]. WB-DWI studies are generally acquired with a single-shot echo-planar imaging (EPI) sequence which is very efficient and motion-robust, combined with fat suppression to minimise artefacts. EPI is, however, prone to geometric distortion and signal loss, which are the result of phase accumulation in regions of high susceptibility gradient. This is especially pronounced in regions of high susceptibility gradient, notably within the 
head (e.g. base of skull, sinuses), neck, and thorax (e.g. chest wall) [7-9]. Another drawback of conventional WB-DWI techniques is the spinal cord displacement artefact which is often apparent when WB-DWI is viewed as a sagittally reformatted image (as is common in clinical practice) [10]. The different shim settings which are applied for the acquisition of each station result in an apparent spatial displacement at the junction of adjacent stations when reformatted in the sagittal plane.

The presence of distortion, signal loss, and artefacts in WB-DWI studies can hamper evaluation of bone disease and cord compression in myeloma [7, 10,11]. Several techniques have been developed to overcome these issues. The spinal cord displacement artefact can be minimised by applying the shim settings of one station to all subsequent stations [12]. However, this can lead to other image quality issues, such as more pronounced chemical shift artefacts and failure of frequency-selective fat suppression [10]. Distortion and/ or signal loss can be improved by reducing the phase field of view [13-16], reducing the echo spacing [17], using the reverse gradient polarity method [18], or by post-processing [19]. Each of these techniques has been shown to improve EPI image quality, but residual distortion, signal loss and artefacts remain due to B0 inhomogeneity. Slice-specific dynamic shimming is a technique which could improve the limitations of EPI by reducing B0 inhomogeneity [20]. At each station, the prototype integrated slice-specific dynamic shimming method (iShim) used in this work [21] acquires a 2-dimensional gradient-recalled echo sequence before the WB-DWI acquisition, which is used to calculate a field map for each slice. From the field maps, a centre frequency and linear shim terms are determined for each slice. The centre frequency and linear shim terms are then set (dynamically updated) before the acquisition of each EPI slice. This shimming technique is restricted to first-order shimming, with higher order shims set to a constant, patient-independent value, as shim current settling times for higher order shimming would exceed the minimum time between slice acquisitions. In particular, the values of the higher order shim currents are not modified between the acquisition of the field map and the actual scan. The acquired field map, corrected for first-order shim settings, can, however, be used to correct for residual distortion $[19,21]$. This combination of slicespecific shimming and post-processing has an advantage over post-processing alone, which cannot compensate for signal loss [21].

A number of studies have reported improvement in signalto-noise ratio and reduction in geometric distortion at $3 \mathrm{~T}$ for prostate [22], head and neck [8, 9], thyroid [23], breast [24], and bladder [25] for slice-specific dynamic shimming over conventional volume-based shimming. Slice-specific dynamic shimming has also been shown to be effective in reducing distortion, signal loss, and spatial displacement artefacts for WB-DWI at $3 \mathrm{~T}$ [26]. There are, however, few studies investigating the impact of slice-specific shimming at $1.5 \mathrm{~T}$, which represents a significant proportion of clinical scanners installed [27]. While DWI is more prone to distortion, signal loss, and artefacts at $3 \mathrm{~T}$, these drawbacks are also present at $1.5 \mathrm{~T}[12,19,20]$. Therefore, the aim of our study was to investigate the impact of slice-specific shimming with distortion correction post-processing for the reduction of artefacts and signal loss compared to conventional 3D volume shimming for $1.5 \mathrm{~T}$ WB-DWI.

\section{Methods}

\section{Subjects}

Ten healthy volunteers (three male, seven female, median age 34 years, age range 24-38 years) were recruited for the study, following approval by the NRES London Bridge research ethics committee (study number 01/11/12) and providing written informed consent. Additionally, haematology patients undergoing WB-MRI for suspected myeloma were retrospectively identified from our institutional database (scan dates between January 2017 and January 2018). Twenty-four consecutive patients scanned with the conventional 3D volume shim WB-DWI sequence (13 male, 11 female, median age 72 years, age range 49-87) and 24 consecutive patients scanned with the iShim WB-DWI sequence (11 male, 13 female, median age 62 years, age range 28-87) were included. A review board waiver was granted for the analysis of anonymised patient data.

\section{Magnetic resonance imaging}

All subjects were scanned on a 1.5 T MR scanner (MAGNETOM Aera, Siemens Healthcare, Erlangen, Germany, VE11C software). Volunteers and patients were positioned in a head-first, supine position and scanned with a standard protocol consisting of seven axial imaging stations (each consisting of $40 \times 5 \mathrm{~mm}$ contiguous slices), covering from skull vertex to mid-calf. At each imaging station, T1-weighted 3D Dixon FLASH (VIBE), T2-weighted 2D single-shot turbo spin echo (HASTE), and 2D single-shot echo-planar DWI sequences with conventional 3D volume shim and/or with prototype iShim functionality [21] were acquired. All DWI acquisitions were acquired in freebreathing. The MR table was automatically positioned at the magnet isocenter for each station. Apart from the shimming method (either iShim or 3D volume shim, or both, depending on cohort), the DWI acquisition parameters were identical (Table 1). Patients also underwent sagittal 2D T1-weighted and STIR TSE imaging of the spine, as well as coronal T1-weighted pre- and post-contrast VIBE imaging. 
Table 1 Acquisition parameters for in-vivo studies

\begin{tabular}{ll}
\hline Acquisition plane & Axial \\
\hline TR/TE (ms) & $6360 / 67$ \\
FOV $\left(\mathrm{mm}^{2}\right)$ & $500 \times 500$ \\
Acquisition matrix & $128 \times 128$ \\
Slice thickness (mm) & 5 \\
Slice gap (mm) & 0 \\
Number of slices per station & 40 \\
Number of stations & 7 \\
In-plane acceleration & GRAPPA 2, integrated reference \\
& lines: 42 \\
Fat suppression & STIR (TI $=180$ ms) \\
Receiver bandwidth (Hz/pixel) & 2300 \\
Diffusion mode & \\
B values (s/mm ${ }^{2}$ ) & 3 -scan Trace \\
NSA & 50,900 \\
& 2 for $b=50$ s/mm ${ }^{2}$ \\
Diffusion gradient scheme & 5 for $b=900$ s/mm ${ }^{2}$ \\
Dynamic field correction & Monopolar (single refocused spin \\
Composing algorithm & echo diffusion sensitizing) \\
Receiver coils & On \\
& Diffusion \\
Total acquisition time & Head/neck matrix \\
& $2 \times$ body matrix \\
& Spine matrix \\
Peripheral angio matrix & $19: 30($ volume shim) \\
& $23: 30$ (iShim) \\
\hline
\end{tabular}

$T R$ repetition time, $T E$ echo time, FOV field of view, STIR short-tau inversion recovery, $T I$ inversion time, NSA number of signal averages

a 3 -scan trace acquires three orthogonal oblique gradient directions sequentially

${ }^{\mathrm{b}}$ Small increase in imaging time for iShim as a result of additional shimming and frequency adjustment

\section{Image analysis}

Absolute spinal cord displacement artefact was measured in all subjects at each junction between imaging stations 1 and 4 (cervical, thoracic and lumbar junctions), all of which include the spinal cord in most individuals. In each case, the distance was measured from the anterior aspect of the spinal cord on the lower station to the anterior aspect of the spinal cord on the upper station.

A sternum-to-vertebra signal intensity ratio (SIR) was calculated to assess signal loss in a region of high susceptibility gradient. Circular regions of interest (ROIs) of $1 \mathrm{~cm}^{2}$ were drawn over the sternum and a region of relatively low susceptibility gradient (vertebral body), both at the same cranio-caudal position, on a mid-sagittal slice of all volunteer scans. The average signal intensity in these ROIs was recorded and the sternum-to-vertebra SIR was calculated as a ratio of these measurements. A higher SIR corresponds to lower signal loss in the sternum, which may be achieved with improved shimming in this region of high susceptibility gradient. These measurements were performed on volunteer images only since the presence of pathology could bias the results of these measurements in patient studies.

Image quality was graded qualitatively in patients from 1 to $5(1=$ poor, $2=$ unsatisfactory, $3=$ satisfactory, $4=$ very good, $5=$ excellent), and signal intensity discontinuity scores, describing signal intensity discontinuity between adjacent bed positions, were recorded as $1-4,(1=2+$ steps, $2=2$ steps, $3=1$ step, $4=$ no steps) [26].

Spinal cord displacement measurements were carried out three times in total in healthy volunteers: twice by an MR physicist and once by a radiologist, to enable characterisation of the intra- and inter-observer reproducibility of this method.

Signal intensity ratios in volunteers were measured by an MR physicist. Two radiologists with 10 years and 5 years of whole-body MRI experience independently analysed the patient studies. All analysis was performed on sagittal inverted greyscale images reconstructed from axial $b=900 \mathrm{~s} / \mathrm{mm}^{2}$ images (reflecting standard reporting practice at our institution), using the MR scanner manufacturer's post-processing software (syngo.via VB20A; Siemens Healthcare, Erlangen, Germany).

\section{Statistical analysis}

All statistical analysis was carried out using Microsoft Excel 2018. As absolute spinal cord displacement measurements are not normally distributed, the Wilcoxon signed-rank test was used to test whether the spinal cord displacement measured on the iShim DWI sequence was different from the displacement measured on the volume shim DWI sequence in volunteers. Bland-Altman analysis was used to determine the intra- and inter-observer reproducibility of spinal cord displacement measurements. The Shapiro-Wilk test was used to test for normality of SIR measurements. A twotailed paired $t$ test was used to compare the difference in SIRs between the iShim and volume shim DWI sequences in volunteers. The Mann-Whitney $U$ test was used to test the difference in spinal cord displacement, image quality scores, and signal discontinuity scores between iShim and volume 3D shim DWI sequences in patients. The value $p<0.05$ was considered statistically significant in all statistical tests.

\section{Results}

The distribution of spinal cord displacement offsets in the healthy volunteers and patients are presented in Fig. 1. The cord did not extend to the bottom station junction in three volunteers, so the results are shown for seven volunteers for this region. The iShim sequence demonstrated a significant 

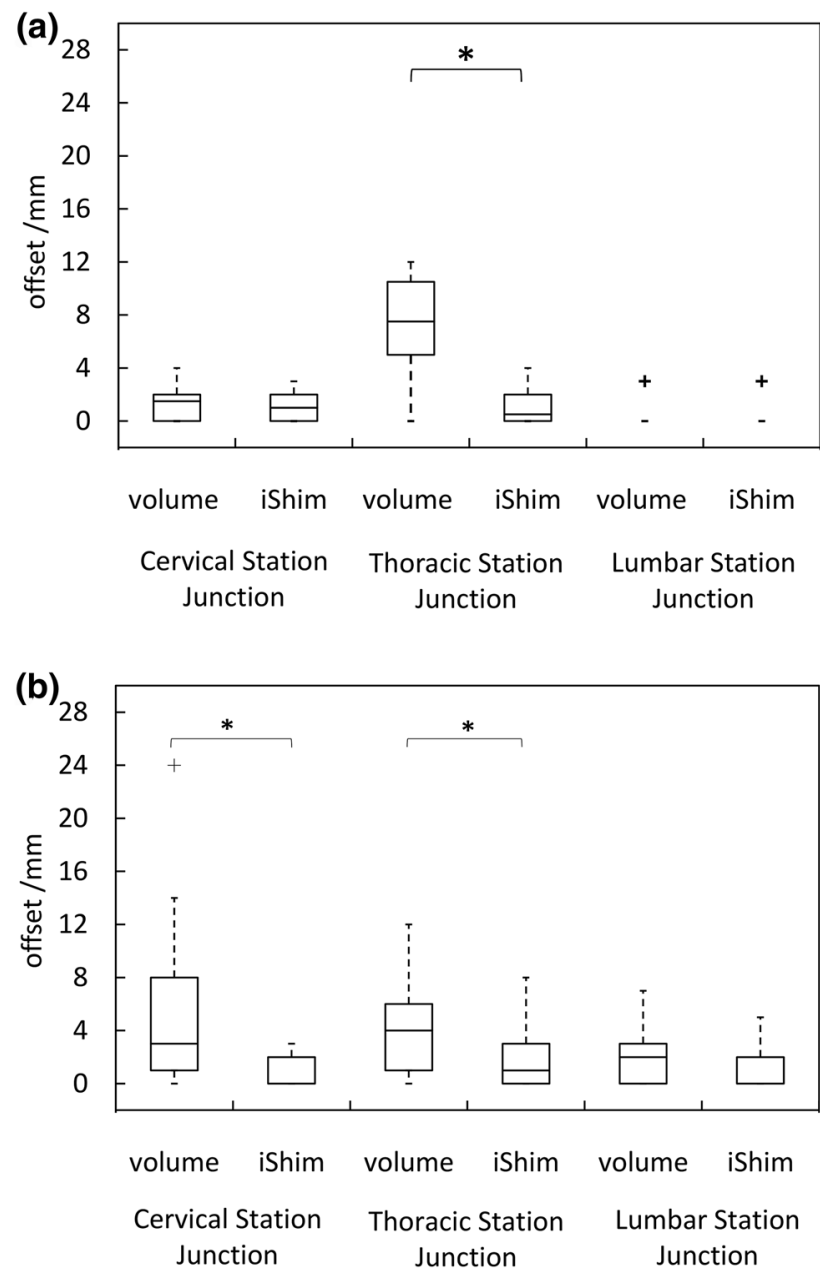

Fig. 1 Spinal cord displacement offsets measured on sagittally reformatted images composed from axially acquired $b=900 \mathrm{~s} / \mathrm{mm}^{2}$ images. a Boxplots showing offset in positions of spinal cord at the cervical and thoracic station junctions in ten healthy volunteers. Displacement at the lumbar station junction was measured in seven volunteers as the spinal cord did not extend to the lumbar station junction in three volunteers. The values for the median and quartiles 1 and 3 for this station were equal to zero for this station, resulting in no 'box' for this plot. b Boxplots showing offset in positions of spinal cord at cervical, thoracic and lumbar station junctions in 24 patients scanned with the conventional 3D volume shim WB-DWI sequence and 24 patients scanned with the iShim WB-DWI sequence. Offsets were measured on sagittally reformatted images composed from axially acquired $b=900 \mathrm{~s} / \mathrm{mm}^{2}$ images

reduction in spinal cord displacement compared with volume shim at the thoracic junction in volunteers $(p<0.05)$ and at the cervical junction $(p<0.05)$ and thoracic junction $(p<0.05)$ in patients. The maximum spinal cord displacement artefact was reduced from 12 to $4 \mathrm{~mm}$ using volume shim and iShim, respectively, in volunteers and from 24 to $8 \mathrm{~mm}$ using volume shim and iShim respectively in patients. Figure 2 shows an exemplary case demonstrating the reduction of spinal cord displacement at the thoracic junction on images of a volunteer. Markedly reduced distortion in the head and cervical spine stations was also noted on the iShim image. Bland-Altman plots of the intra- and interobserver reproducibility of the spinal cord displacement artefact measurement in volunteers are shown in Fig. 3. The Bland-Altman mean difference and limits of agreement for intra- and inter-observer reproducibility are shown in Tables 2 and 3, respectively. The sternum-to-vertebra SIR was significantly increased in the iShim images compared with matched volume shim images (Fig. 4, $p<0.05$, paired $t$ test). The distribution of image quality and signal intensity discontinuity scores are shown in Fig. 5. There were no significant differences in image quality or signal intensity discontinuity scores between iShim and volume shim patient data sets ( $p>0.05$, Mann-Whitney $U$ test). Figure 6a shows an example of spinal cord displacement artefact at the region of malignant thoracic cord compression in a patient with myeloma.

\section{Discussion}

In this study, we compared iShim against volume shim for 1.5 T WB-DWI. Spinal cord displacement and susceptibility-related signal loss were lower using the iShim sequence compared to the volume shim sequence. Qualitative assessment of patient images showed no significant differences in image quality and signal intensity discontinuity scores. Spinal cord displacement artefact is caused by a discrepancy between the centre frequencies and shim terms of adjacent slices at the interface between two stations. Using the conventional 3D volume shimming technique, these values can vary quite significantly between adjacent slices at a station junction, since they are optimised for a whole station. With iShim, the centre frequency and shim terms are optimised for each slice, so the discrepancy between these values for any two adjacent slices is likely to be small.

The displacements measured in our study were lower than comparable measurements at $3 \mathrm{~T}$. In one $3 \mathrm{~T}$ study [26], the maximum measured displacements, excluding outliers, were $20 \mathrm{~mm}$ and $16 \mathrm{~mm}$ using volume shim and iShim, respectively, in comparison to $14 \mathrm{~mm}$ and $8 \mathrm{~mm}$ in our study. This larger displacement measured at $3 \mathrm{~T}$ is likely caused by an increased B0 inhomogeneity at this field strength. Our study also employed retrospective field map based distortion correction (which was not used for the $3 \mathrm{~T}$ study), although a small study in ten patients did not demonstrate a significant reduction in spinal cord displacement artefact compared with slice-specific shimming alone (see Supplementary Material, Table 1). In clinical practice, it is desirable to reduce the presence of any significant artefact, the presence of which may result in reduced diagnostic confidence, increased reporting times, a need for repeat imaging, or, in the worst case, a misdiagnosis. 


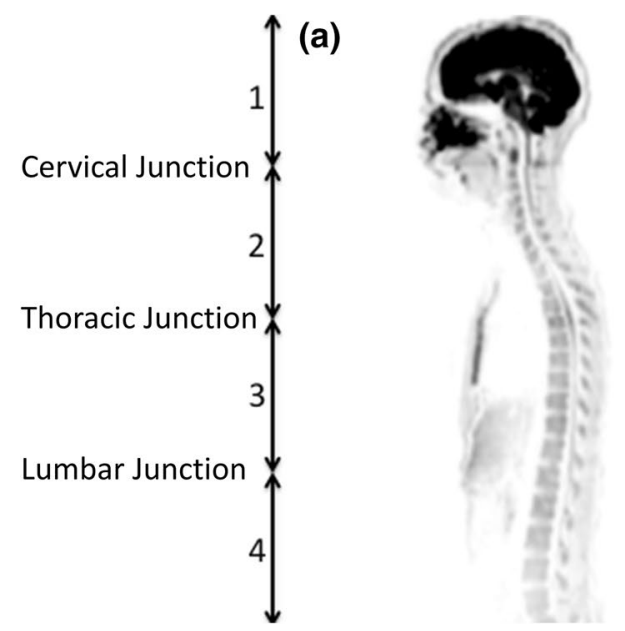

(b)

Fig. 2 Sagittally reformatted composed images of volunteer study. a $b=900 \mathrm{~s} / \mathrm{mm}^{2}$ images acquired using iShim. b $b=900 \mathrm{~s} / \mathrm{mm}^{2}$ images acquired using volume shim. c T2-weighted HASTE image as an anatomical reference image with no significant distortion. Locations

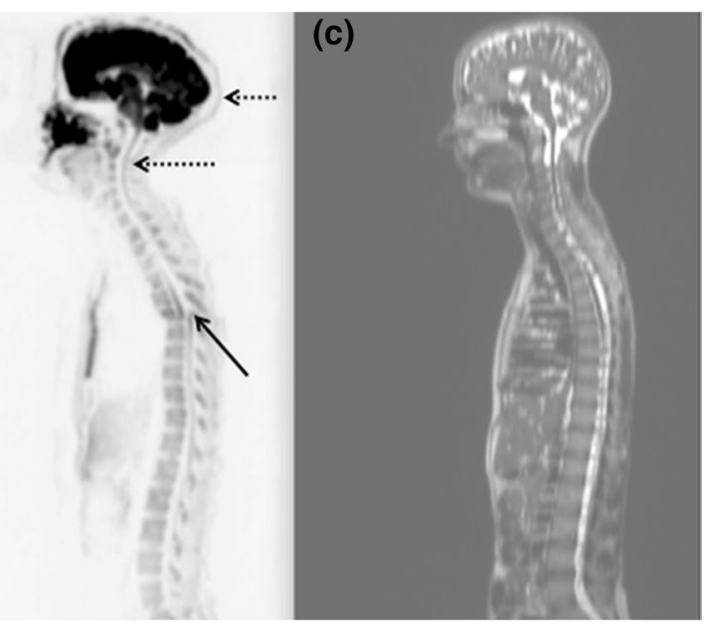

of stations (1-4) are shown by arrows on left-hand side. Solid arrow shows offset in positions of spinal cord at the thoracic station junction in diffusion-weighted images acquired using volume shim. Dashed arrows show distortion of spinal cord and head in upper station
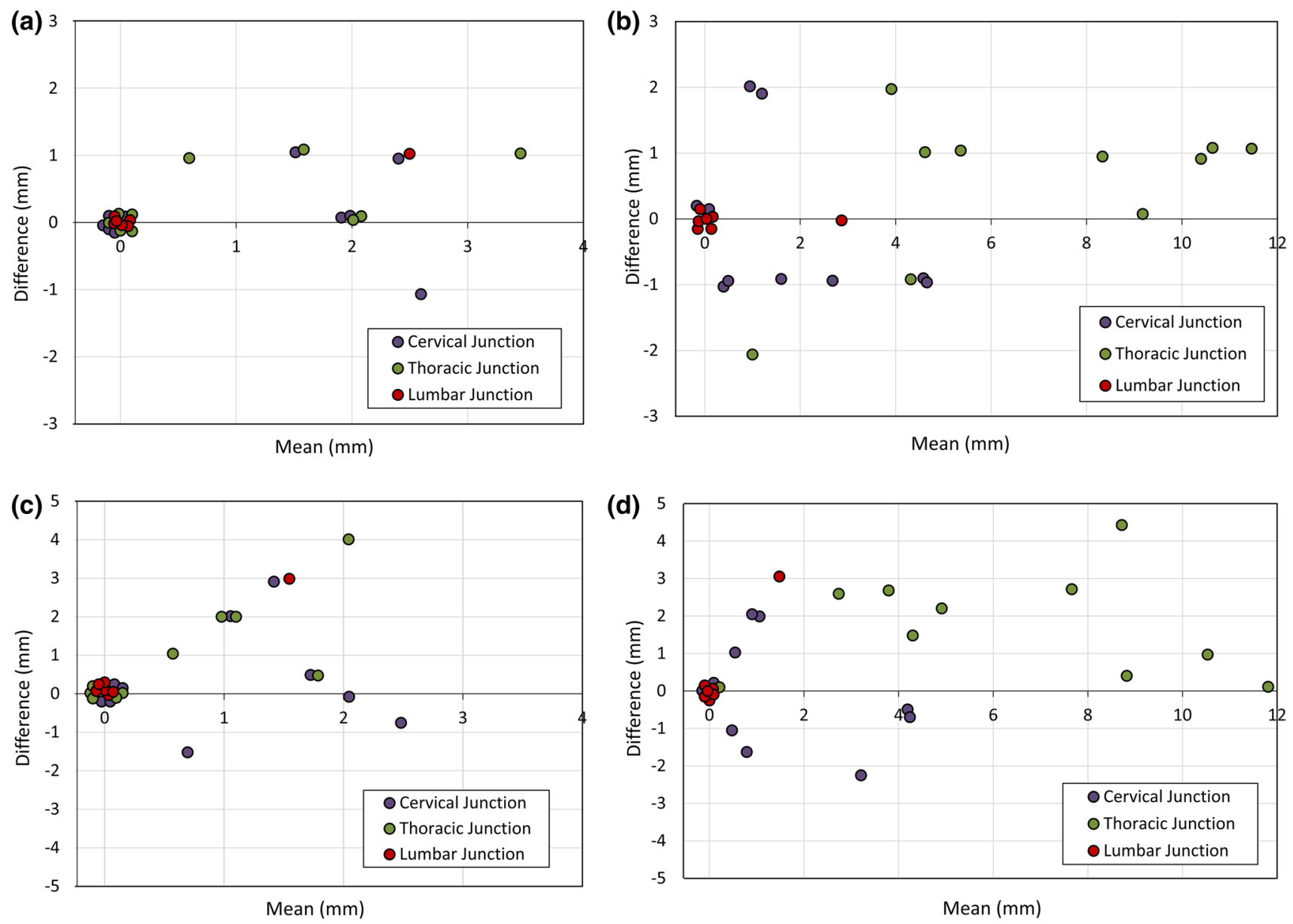

Fig. 3 Bland-Alman plots showing intra-observer reproducibility (a, b) and inter-observer reproducibility (c, d) of spinal cord displacement artefact measurements in healthy volunteers,using the iShim

shim DWI sequence $(\mathbf{a}, \mathbf{c})$ and the volume shim sequence $(\mathbf{b}, \mathbf{d})$. Overlapping points have been marginally offset for clarity 
Table 2 Bland-Altman mean difference and limits of agreement in millimetres for the intra-observer reproducibility of spinal cord displacement artefact measurements in volunteers

\begin{tabular}{lcc}
\hline & iShim & Volume shim \\
\hline Cervical station junction & $0.1 \pm 1.1$ & $0 . \pm 2.6$ \\
Thoracic station junction & $-0.3 \pm 1.0$ & $0.5 \pm 2.4$ \\
Lumbar station junction & $0.14 \pm 0.75$ & $0.0 \pm 0.0$ \\
\hline
\end{tabular}

Table 3 Bland-Altman mean difference and limits of agreement in millimetres for the inter-observer reproducibility of spinal cord displacement artefact measurements in volunteers

\begin{tabular}{llc}
\hline & iShim & Volume shim \\
\hline Cervical station junction & $0.3 \pm 2.5$ & $-0.1 \pm 2.8$ \\
Thoracic station junction & $0.9 \pm 2.6$ & $1.8 \pm 2.8$ \\
Lumbar station junction & $0.4 \pm 2.2$ & $0.4 \pm 2.2$ \\
\hline
\end{tabular}

Limits of agreement are calculated as $1.96 \times$ standard deviation

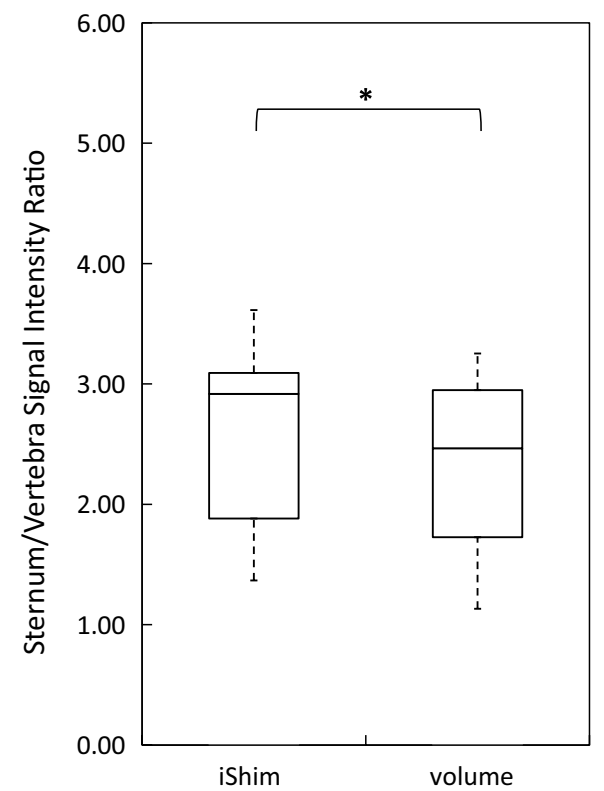

Fig. 4 Boxplots showing sternum-to-vertebra signal intensity ratio in ten healthy volunteers. Signal intensity was measured on $1 \mathrm{~cm}^{2}$ circular ROIs drawn on sagittally reformatted images composed from axially-acquired $b=900 \mathrm{~s} / \mathrm{mm}^{2}$ images

The $3 \mathrm{~T}$ WB-DWI iShim study also reached different conclusions compared with the current study with regards to image quality scores and signal intensity discontinuities. At $3 \mathrm{~T}$, image quality scores showed an increase using iShim and signal intensity discontinuities decreased with iShim. This disparity between the two studies can be attributed to a lower degree of distortion and signal loss of DWI measurements at $1.5 \mathrm{~T}$.
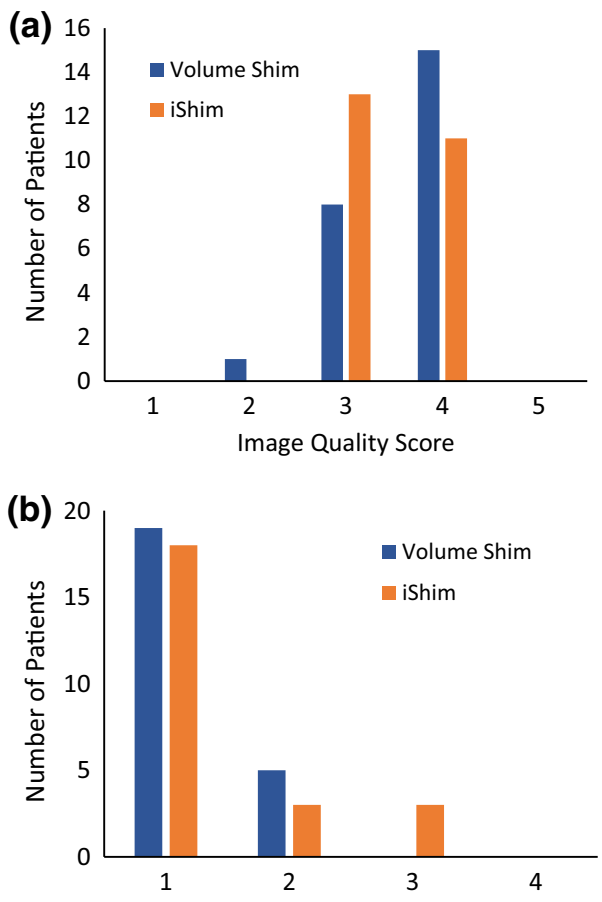

Signal Intensity Discontinuity Score

Fig. 5 Qualitative assessment of images. a Image quality scores for patients. $1=$ poor, $2=$ unsatisfactory, $3=$ satisfactory, $4=$ very good, $5=$ excellent. b Signal intensity discontinuity scores for patients. $1=2+$ steps, $2=2$ steps, $3=1$ step, $4=0$ steps

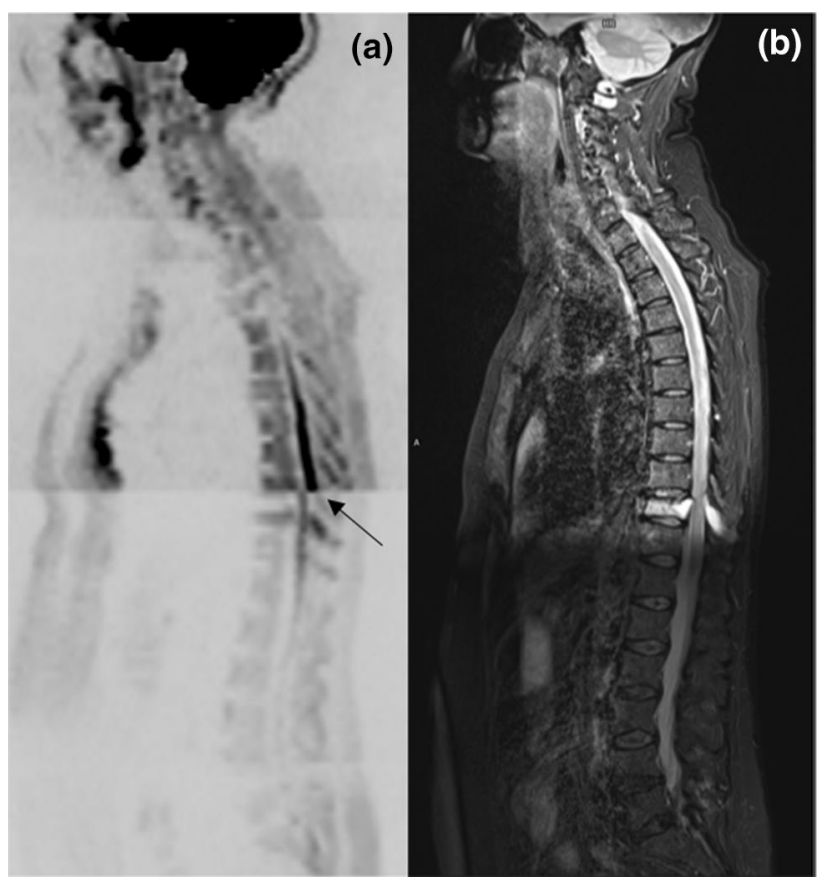

Fig. 6 Sagittally reformatted composed images of patient study. a $b=900 \mathrm{~s} / \mathrm{mm}^{2}$ images acquired using volume shim. b T2-weighted HASTE. Arrow indicates the spinal cord displacement artefact at the region of malignant cord compression 
It was not possible to compare absolute signal intensity values in the sternum due to different post-processing steps resulting in different signal intensity scaling between iShim and volume shim images. Therefore, we used a relative assessment of signal loss in a region of high susceptibility gradient (sternum) to a region of lower susceptibility gradient (vertebra). A comparison of the signal intensity ratios measured with the two DWI sequences indicate a reduction of susceptibility-related signal loss in the sternum with the iShim sequence. Susceptibility-related signal losses could affect interpretation in cases of sternal disease. Reduced signal loss with iShim has also been demonstrated in other regions of the body with high susceptibility gradient, such as the neck [8].

In terms of workflow, dynamic shimming and retrospective distortion correction are integrated into the iShim sequence, maintaining a streamlined workflow, with a small increase in imaging time to allow for additional shimming and frequency adjustment. This has an advantage over other solutions, such as applying a fixed centre frequency across all stations, which require greater operator input.

Our study has some limitations. Due to patient comfort and scan-time restrictions, only a single DWI sequence was acquired in each patient. Therefore, the impact of the artefact on interpretation was limited as we could not directly compare the two sequences in each patient. Additionally, our study evaluated the performance of iShim as one vendor's solution to reduce the distortion and signal loss associated with WB-DWI acquisitions. Therefore, the impact of dynamic shimming cannot be compared across different vendors.

\section{Conclusion}

With the recommendation of WB-MRI as a first line investigation in a number of guidelines and an associated rise in the number of studies performed, image quality optimisation is essential, particularly as many of these patients may undergo serial studies, e.g. for response assessment. The results of our study demonstrate that the iShim technique can reduce spinal cord displacement artefacts and signal loss in WB-DWI scans at $1.5 \mathrm{~T}$, which is currently the most commonly-used field strength for WB-MRI studies. There were no differences in terms of image quality scores and signal intensity discontinuities with the iShim technique compared to a conventional volume shim technique.

Acknowledgements The Myeloma Imaging Research Group at Guy's and St Thomas' Hospital and King's College London include the following (in alphabetical order): Dr Ashik Amlani, Ms Joanna Bell, Professor Gary JR Cook, Dr Geoff Charles-Edwards, Dr Isabel Dregely, Dr Inas El-Najjar, Miss Renyang Gu, Dr Ulrike Haberland, Dr Majid
Kazmi, Dr Chistian Kelly-Morland, Dr Sarah Natas, Dr Ana Pascoal, Mr John Spence, Dr Matthew Streetly, Dr Manil Subesinghe, Dr Hema Verma, Dr Zaid Viney. The authors acknowledge financial support from the King's College London/University College London Comprehensive Cancer Imaging Centres funded by Cancer Research UK and Engineering and Physical Sciences Research Council in association with the Medical Research Council and the Department of Health (Grant number C1519/A16463), and the Wellcome Trust EPSRC Centre for Medical Engineering at King's College London (Grant number WT203148/Z/16/Z).

Author contributions MS: study conception and design, acquisition of data, analysis and interpretation of data, drafting of manuscript, and critical revision. WJ: study conception and design, acquisition of data, analysis and interpretation of data, drafting of manuscript, and critical revision. WO: study conception and design, acquisition of data, analysis and interpretation of data, drafting of manuscript, and critical revision. C-EG: study conception and design, acquisition of data, and critical revision. BJ: acquisition of data and critical revision. NR: study conception and design, acquisition of data, and critical revision. SA: study conception and design and critical revision. KB: study conception and design and critical revision. SM: acquisition of data and critical revision. GV: study conception and design, acquisition of data, analysis and interpretation of data, drafting of manuscript, and critical revision.

Funding This study was funded by Cancer Research UK and Engineering and Physical Sciences Research Council in association with the Medical Research Council and the Department of Health (Grant number C1519/A16463), and the Wellcome Trust EPSRC Centre for Medical Engineering at King's College London (Grant number WT203148/Z/16/Z).

\section{Compliance with ethical standards}

Conflict of interest Dr. Radhouene Neji, Dr. Alto Stemmer and Dr. Berthold Kiefer are employees of Siemens Healthcare.

Ethical approval All procedures performed in studies involving human participants were in accordance with the ethical standards of the institutional and/or national research committee and with the 1964 Helsinki declaration and its later amendments or comparable ethical standards.

Informed consent Informed consent was obtained from all healthy volunteers included in the study. A review board waiver was granted for the analysis of anonymised patient data.

Open Access This article is licensed under a Creative Commons Attribution 4.0 International License, which permits use, sharing, adaptation, distribution and reproduction in any medium or format, as long as you give appropriate credit to the original author(s) and the source, provide a link to the Creative Commons licence, and indicate if changes were made. The images or other third party material in this article are included in the article's Creative Commons licence, unless indicated otherwise in a credit line to the material. If material is not included in the article's Creative Commons licence and your intended use is not permitted by statutory regulation or exceeds the permitted use, you will need to obtain permission directly from the copyright holder. To view a copy of this licence, visit http://creativecommons.org/licenses/by/4.0/. 


\section{References}

1. Rajkumar SV, Dimopoulos MA, Palumbo A, Blade J, Merlini G, Mateos M-V, Kumar S, Hillengass J, Kastritis E, Richardson P, Landgren O, Paiva B, Dispenzieri A, Weiss B, LeLeu X, Zweegman S, Lonial S, Rosinol L, Zamagni E, Jagannath S, Sezer O, Kristinsson SY, Caers J, Usmani SZ, Lahuerta JJ, Johnsen HE, Beksac M, Cavo M, Goldschmidt H, Terpos E, Kyle RA, Anderson KC, Durie BGM, Miguel JFS (2014) International Myeloma Working Group updated criteria for the diagnosis of multiple myeloma. Lancet Oncol 15:e538-e548

2. National Institute for Health and Care Excellence (2016) Myeloma: diagnosis and management. NICE guideline (NG35). http:// www.nice.org.uk/guidance/ng35

3. Takahara T, Imai Y, Yamashita T, Yasuda S, Nasu S, Cauteren MV (2004) Diffusion weighted whole body imaging with background body signal suppression (DWIBS): technical improvement using free breathing, STIR and high resolution 3D display. Radiat Med 22:8

4. Gu J, Chan T, Zhang J, Leung AYH, Kwong Y-L, Khong P-L (2011) Whole-body diffusion-weighted imaging: the added value to whole-body MRI at initial diagnosis of lymphoma. Am J Roentgenol 197:W384-W391

5. Pawlyn C, Fowkes L, Otero S, Jones JR, Boyd KD, Davies FE, Morgan GJ, Collins DJ, Sharma B, Riddell A, Kaiser MF, Messiou C (2016) Whole-body diffusion-weighted MRI: a new gold standard for assessing disease burden in patients with multiple myeloma? Leukemia 30:1446-1448

6. Takenaka D, Ohno Y, Matsumoto K, Aoyama N, Onishi Y, Koyama H, Nogami M, Yoshikawa T, Matsumoto S, Sugimura K (2009) Detection of bone metastases in non-small cell lung cancer patients: comparison of whole-body diffusion-weighted imaging (DWI), whole-body MR imaging without and with DWI, whole-body FDG-PET/CT, and bone scintigraphy. J Magn Reson Imaging 30:298-308

7. Buchbender C, Hartung-Knemeyer V, Beiderwellen K, Heusch P, Kühl H, Lauenstein TC, Forsting M, Antoch G, Heusner TA (2013) Diffusion-weighted imaging as part of hybrid PET/MRI protocols for whole-body cancer staging: does it benefit lesion detection? Eur J Radiol 82:877-882

8. Gatidis S, Graf H, Weiß J, Stemmer A, Kiefer B, Nikolaou K, Notohamiprodjo M, Martirosian P (2017) Diffusion-weighted echo planar MR imaging of the neck at $3 \mathrm{~T}$ using integrated shimming: comparison of MR sequence techniques for reducing artifacts caused by magnetic-field inhomogeneities. Magn Reson Mater Phy 30:57-63

9. Walter SS, Liu W, Stemmer A, Martirosian P, Nikolaou K, Notohamiprodjo M, Gatidis S (2017) Combination of integrated dynamic shimming and readout-segmented echo planar imaging for diffusion weighted MRI of the head and neck region at 3 Tesla. Magn Reson Imaging 42:32-36

10. Koh D-M, Blackledge M, Padhani AR, Takahara T, Kwee TC, Leach MO, Collins DJ (2012) Whole-body diffusion-weighted MRI: tips, tricks, and pitfalls. Am J Roentgenol 199:252-262

11. Messiou C, Hillengass J, Delorme S, Lecouvet FE, Moulopoulos LA, Collins DJ, Blackledge MD, Abildgaard N, Østergaard B, Schlemmer H-P, Landgren O, Asmussen JT, Kaiser MF, Padhani A (2019) Guidelines for acquisition, interpretation, and reporting of whole-body mri in myeloma: myeloma response assessment and diagnosis system (MY-RADS). Radiology 291:5-13

12. Li S, Sun F, Jin Z, Xue H, Li M (2007) Whole-body diffusionweighted imaging: technical improvement and preliminary results. J Magn Reson Imaging 26:1139-1144
13. Griswold MA, Jakob PM, Chen Q, Goldfarb JW, Manning WJ, Edelman RR, Sodickson DK (1999) Resolution enhancement in single-shot imaging using simultaneous acquisition of spatial harmonics (SMASH). Magn Reson Med 41:1236-1245

14. Bammer R, Keeling SL, Augustin M, Pruessmann KP, Wolf R, Stollberger R, Hartung H-P, Fazekas F (2001) Improved diffusionweighted single-shot echo-planar imaging (EPI) in stroke using sensitivity encoding (SENSE). Magn Reson Med 46:548-554

15. Rieseberg S, Frahm J, Finsterbusch J (2002) Two-dimensional spatially-selective RF excitation pulses in echo-planar imaging. Magn Reson Med 47:1186-1193

16. Rosenkrantz AB, Chandarana H, Pfeuffer J, Triolo MJ, Shaikh MB, Mossa DJ, Geppert C (2015) Zoomed echo-planar imaging using parallel transmission: impact on image quality of diffusion-weighted imaging of the prostate at 3T. Abdom Imaging 40:120-126

17. Porter DA, Heidemann RM (2009) High resolution diffusionweighted imaging using readout-segmented echo-planar imaging, parallel imaging and a two-dimensional navigator-based reacquisition. Magn Reson Med 62:468-475

18. Holland D, Kuperman JM, Dale AM (2010) Efficient correction of inhomogeneous static magnetic field-induced distortion in echo planar imaging. NeuroImage 50:175-183

19. Jezzard P, Balaban R (1995) Correction for geometric distortion in echo planar images from B0 field variations. Magn Reson Med 34:65-73

20. Morrell G, Spielman D (1997) Dynamic shimming for multi-slice magnetic resonance imaging. Magn Reson Med 38:477-483

21. Stemmer A, Kiefer B (2015) Combination of integrated slicespecific dynamic shimming and pixel-wise unwarping of residual EPI distortions. Proc Int Soc Mag Reson Med 23:3729

22. Stocker D, Manoliu A, Becker AS, Barth BK, Nanz D, Klarhöfer M, Donati OF (2018) Image quality and geometric distortion of modern diffusion-weighted imaging sequences in magnetic resonance imaging of the prostate. Invest Radiol 53:200-206

23. Chen L, Sun P, Hao Q, Yin W, Xu B, Ma C, Stemmer A, Fu C, Wang M, Lu J (2018) Diffusion-weighted MRI in the evaluation of the thyroid nodule: Comparison between integrated-shimming EPI and conventional 3D-shimming EPI techniques. Oncotarget 9(26209-26216):24

24. Lee S-K, Tan ET, Govenkar A, Hancu I (2014) Dynamic slicedependent shim and center frequency update in $3 \mathrm{~T}$ breast diffusion weighted imaging: dynamic shim update for breast DWI. Magn Reson Med 71:1813-1818

25. Li H, Liu L, Shi Q, Stemmer A, Zeng H, Li Y, Zhang M (2017) Bladder cancer: detection and image quality compared among iShim, RESOLVE, and ss-EPI diffusion-weighted MR imaging with high b value at 3.0 T MRI. Medicine (Baltimore) 96:e9292

26. Zhang H, Xue H, Stemmer A, Hui L, Kannengiesser S, Kiefer B, Jin Z (2015) Integrated shimming improves lesion detection in whole-body diffusion-weighted examinations of patients with plasma disorder at $3 \mathrm{~T}$. Invest Radiol 56:297-305

27. The Royal College of Radiologists (2017) Magnetic resonance imaging (MRI) equipment, operations and planning in the NHS report from the clinical imaging board. The Royal College of Radiologists, London

Publisher's Note Springer Nature remains neutral with regard to jurisdictional claims in published maps and institutional affiliations. 


\section{Affiliations}

\section{Sarah McElroy ${ }^{1}$ (D) . Jessica M. Winfield ${ }^{1} \cdot$ Olwen Westerland $^{1} \cdot$ Geoff Charles-Edwards $^{1} \cdot$ Joanna Bell $^{1}$. Radhouene Neji ${ }^{2}$. Alto Stemmer ${ }^{3}$ - Berthold Kiefer ${ }^{3} \cdot$ Matthew Streetly $^{4}$ - Vicky Goh ${ }^{1,5}$}

Jessica M. Winfield

jessica.winfield@icr.ac.uk

Olwen Westerland

olwen.westerland@kcl.ac.uk

Joanna Bell

joanna.bell@kcl.ac.uk

Radhouene Neji

radhouene.neji@siemens.com

Alto Stemmer

alto.stemmer@siemens.com

Berthold Kiefer

Berthold.kiefer@siemens.com

Matthew Streetly

Matthew.Streetly@gstt.nhs.uk
Vicky Goh

vicky.goh@kcl.ac.uk

1 Clinical Imaging and Medical Physics, Guy's and St Thomas' Hospital, London, UK

2 MR Research Collaborations, Siemens Healthcare, Frimley, UK

3 MR Application Predevelopment, Siemens Healthcare, Erlangen, Germany

4 Clinical Haematology, Guy's and St Thomas' NHS Foundation Trust, London, UK

5 Department of Cancer Imaging, School of Biomedical Engineering and Imaging Sciences, King's College London, London, UK 